\title{
MEASURING FUNCTIONAL HEALTH LITERACY IN HUNGARY: VALIDATION OF S-TOFHLA AND CHEW SCREENING QUESTIONS
}

\author{
Lilla Náfrádi ${ }^{1}$, Orsolya Papp-Zipernovszky², Peter J. Schulz' ${ }^{1}$, Márta Csabai ${ }^{2}$ \\ ${ }^{1}$ Institute of Communication and Health, University of Lugano, Lugano, Switzerland \\ ${ }^{2}$ Institute of Psychology, University of Szeged, Szeged, Hungary
}

\begin{abstract}
SUMMARY
Objectives: The first efforts to measure health literacy have recently started in Hungary, thus there remains a need for tools that can be effectively used in the clinical setting. The goal of the present study was two-fold: to validate tools for measuring functional health literacy in Hungary using the Short Test of Functional Health Literacy (S-TOFHLA) and the Chew screening measure, and to provide an overview of the health literacy level of the Hungarian population.

Methods: The original English versions of both instruments were translated following the principles of cultural adaptation and standardized translation methods. The measures were administered to a random sample $(\mathrm{N}=302)$ that was close to representative of the Hungarian population regarding age, gender and educational background. The Newest Vital Sign functional health literacy test and numerous socio-demographic variables (such as age, gender, education and income) were also administered to test convergent validity.

Results: The Hungarian version of the S-TOFHLA and the Chew questions showed adequate internal consistency. Lower functional health literacy scores showed the expected association with known predictors of health literacy: higher age, male gender and lower education. Especially people above 65 years of age and individuals with a low level of educational attainment or being chronically ill are vulnerable to have marginal health literacy.

Conclusion: The Hungarian version of the S-TOFHLA is a valid and reliable measure of health literacy. Moreover, the Hungarian version of the Chew screening questions provides a valid self-reported assessment, which is particularly useful to rapidly detect patients with inadequate health literacy in hospitals. It is expected that these health literacy measurements will be used for not only scientific purposes, but also serve as tools for developing public health policy, especially health education and campaigns reducing potential health disparities in Hungary.
\end{abstract}

Key words: health literacy, measurement, validation, S-TOFHLA, Chew screening measure

Address for correspondence: Lilla Nárádi, Institute of Communication and Health, University of Lugano, Via Giuseppe Buffi 13, 6904 Lugano, Switzerland. E-mail: nafradi.lilla@gmail.com

https://doi.org/10.21101/cejph.a4885

\section{INTRODUCTION}

Health literacy can be conceptualized as "the degree to which individuals have the capacity to obtain, process, and understand basic health information and services needed to make appropriate health decisions" (1). There is a great deal of evidence indicating the role of health literacy in many patient outcomes, such as hospitalization and global measures of health (2) and various health behaviours, such as medication adherence (3). Inadequate literacy is a barrier to chronic disease knowledge and self-care, for example in case of asthma patients (4).

There are numerous tools measuring functional health literacy, focusing on basic literacy and numeracy skills. The most widely used instruments are the Rapid Estimate of Functional Health Literacy (REALM) (5), the Test of Functional Health Literacy (TOFHLA) (6) and the Short Test of Functional Health Literacy (S-TOFHLA) (7). The S-TOFHLA is a performance-based measure, which is relatively quick to administer (it takes approximately 12 minutes), consisting of two reading passages and a numeracy part (7). The S-TOFHLA is validated in the following languages:
German, French, Italian (8), Spanish (7), Brazilian Portuguese (9), Hebrew (10), Serbian (11), Turkish (12) and Mandarin (13). The Chew screening questions is also a self-reported functional health literacy measure consisting of three questions. It provides a rapid way to detect patients with inadequate health literacy, therefore, appropriate for clinical setting (14).

Regarding the socio-demographic predictors of health literacy, low educational attainment $(8,10,11,15)$, high age $(8,10,11,15)$ and low income $(10,11)$ are associated with lower health literacy across countries. The findings about gender as an antecedent of health literacy are ambiguous: some studies report that males (10) and according to other studies females $(16,17)$ have higher health literacy level, while other researchers found no relationship (8).

Health literacy is a quickly developing research field, which has the longest tradition in the United States (18). However, recently Europe has developed a multinational research agenda on health literacy (15). The European Health Literacy Survey (HLS-EU) was conducted in eight countries: Austria, Bulgaria, Germany, Greece, Ireland, the Netherlands, Poland and Spain (15). In Hungary, the HLS-EU was carried out involving a 
representative Hungarian sample in 2015. The survey measured self-perceived difficulties in four information processing-related functions: accessing, understanding, appraising, and applying medical information $(15,19)$. The outcomes of the Hungarian survey showed that while the proportion of respondents achieving excellent level was low; the percentage of those receiving inadequate health literacy scores was very high compared to the international standards $(20,21)$. Overall, $20 \%$ of the Hungarian sample fell into inadequate, $32 \%$ to problematic, $38 \%$ to sufficient, and only $11 \%$ into the excellent health literacy category, based on the cumulative health literacy index. The Hungarian average health literacy level fell into a problematic category, similarly to Austria and Spain, only the results in Bulgaria indicated more problems than in Hungary in this regard $(20,21)$. Beside the selfreported health literacy measure, the researchers also applied the Newest Vital Sign (NVS) (22) which indicated that almost onethird of the Hungarian population is below the adequate health literacy level $(20,21)$.

Concerning the operationalization, performance-based tests (such as the S-TOFHLA) provide an accurate and objective assessment of the respondent's health literacy level, but their administration is time consuming and requires the presence of an interviewer (12). On the other hand, self-reported questionnaires, such as the Chew screening questions, can be filled in alone, but are subject to more bias, e.g. social desirability (23). Depending on the context and the goal of the examination, either performance-based or self-reported measures can be more suitable; this study therefore aimed to validate both types of health literacy measurement in Hungary. Moreover, while the HLS-EU cumulative health literacy index is based on self-reported data; the S-TOFHLA provides an objective index for international comparison.

The goal of the present study was to validate the S-TOFHLA and the Chew screening questions in Hungarian. Therefore, we measured health literacy among the Hungarian citizens and examined its associations with socio-demographic variables. In addition, we also evaluated the convergent validity of the tools by examining the relationship between the S-TOFHLA, the Chew screening questions as well as the Newest Vital Sign measure.

\section{MATERIALS AND METHODS}

\section{Original Health Literacy Measures}

The S-TOFHLA (7) is a performance-based functional health literacy measure, which consists of a reading comprehension and a numeracy section. The reading comprehension section has two passages (the first is about an upper gastrointestinal screening and the second is about the patients' rights and responsibilities) adding up to 36 cloze items with multiple-choice options. The respondent has to choose from four answer options of meaning-carrying words that are left blank. The former passage equals to the reading comprehension level, which should be obtained at a 4th grade level, the latter corresponds to a 10th grade level. The reading comprehension section has to be filled in by the respondents and it has a 7-minute time constraint: the respondents overrunning the limit are stopped or their answers are not registered. The numeracy section is administered by the researchers. It consists of 4 numerical tasks: in two cases the respondents have to find the relevant information in a patient information leaflet, one is about judging the correct blood glucose level, and finally participants have to interpret a medical appointment card.

The Chew screening questions (14) is a self-reported measure of functional health literacy consisting of three questions for detecting patients with inadequate or marginal health literacy.

The Newest Vital Sign (22) is a nutrition label that is accompanied by six questions referring to the calories, fat and carbohydrate intake as well as of detecting potential allergens among the ingredients.

\section{Translation and Cultural Adaptation of Tools}

We used a forward and back-translation procedure in order to create a conceptually equivalent Hungarian version of the two measures to the original English versions. The original STOFHLA (7) and Chew screening questions (14) were translated following the principles of cultural adaptation (24). First, an official translator and a bilingual layperson translated the measures independently to Hungarian. Two independent bilingual persons back translated these to check for any inconsistencies. A Hungarian native language teacher approved that the difficulty of the final version of the S-TOFHLA reading passages corresponded to the 4th grade and the 10th grade educational level, respectively.

The Hungarian version of the S-TOFHLA reading comprehension section had to be modified in two cases based on the structure of the sentences and the part of speech compared to the English version. To find the Hungarian equivalent of the four answer options, we took into consideration the similarity of the length of the words in the original test, their rhyming as well as the frequency of the words. The aim was to provide a Hungarian version as conceptually close as possible to the original. The structures of the Hungarian S-TOFHLA and the Chew screening questions correspond completely to the original English versions. However, some minor changes in the content of the S-TOFHLA had to be made due to the difference between the Hungarian and the American healthcare system.

\section{Study Population and Interview Procedure}

The data collection was conducted in collaboration with the Institute of Psychology, University of Szeged, Hungary. Data collection took place between June and October 2015 with convenience sampling, and November-December 2015 with selecting males above 50 years of age with low educational attainment in order to achieve a representative sample of the Hungarian population in terms of age, gender and educational background. The data collection took place in various places: in cafés, hospitals and retirement homes in Hungary. Ethical approval was obtained from the Psychology Ethical Committee of the Universities in Hungary and the ethical committee of the hospital. The sample consists of 302 participants, who are above 20 years of age, Hungarian native speakers and having correct or corrected vision.

After informed consent was obtained from each participant, the test battery consisted of two parts. The researchers administered the respondents' answers for certain questions; the other part was a paper-pencil questionnaire filled in by the participants. The questionnaire consisted of the following instruments: Chew screening questions (14), S-TOFHLA (7), General Self-efficacy 
Scale (25), Newest Vital Sign (22), Health Empowerment Scale that is an adapted version of the Psychological Empowerment Questionnaire (26), Brief Sensation Seeking Scale - 8 (27), and socio-demographic data inventory. The NVS applied in the present article was translated into Hungarian by the authors, although simultaneously another research group prepared another Hungarian version of the NVS $(20,21)$. In the present study, we report solely the outcomes based on the Chew screening questions, STOFHLA, Newest Vital Sign, and socio-demographic variables.

\section{Scoring and Data Analysis}

Participants were categorized into three mutually exclusive groups based on the S-TOFHLA reading comprehension scores: inadequate, marginal and adequate health literacy groups. The scoring system for the S-TOFHLA was based on the Baker et al.'s recommendations: when scoring the reading comprehension part, all the correct answers were assigned one point, and all the incorrect or left blank answers were assigned zero. Therefore, the participants can achieve maximum 36 points. Based on the STOFHLA reading passages the functional health literacy levels are the following: inadequate (0-16), marginal (17-22) and adequate (23-36). In the numeracy part the total score ranges from 0 to 4 (7).

In case of the Chew screening questions (14) each item has an answer option on a Likert-scale ranging from $0-4$, therefore, the summative score ranges from 0 (indicating no problems of health literacy) to 12 (indicating great health literacy-related difficulties). The respondents' points on the first item were reversed.

Data were analyzed using IBM SPSS 21 . The associations between the S-TOFHLA, the Chew screening questions and the NVS were examined with Spearman's rho correlations. The associations between the health literacy scores and well-known socio-demographic antecedents were tested with either independent samples t-test or one-way analysis of variance (ANOVA) with LSD posttest. The explanative power of these antecedents was tested in a linear regression analysis. The significance level of 0.05 were applied as a threshold in the case of all implemented statistical tests.

\section{RESULTS}

\section{S-TOFHLA and Chew Screening Questions Scores of Hungarian Sample}

The study sample is close to representative, as it is within $10 \%$ difference from the Hungarian Statistical Office data regarding the Hungarian population in terms of proportion of gender, age and educational attainment (Table 1).

The S-TOFHLA reading comprehension section showed a very high internal consistency (Cronbach's alpha $=0.955$ ), while the numeracy part had a low internal consistency (Cronbach's alpha $=0.392$ ). The Chew questions showed an adequate internal consistency (Cronbach's alpha $=0.648)$.

In our sample $8.3 \%$ of the participants $(n=25)$ achieved inadequate, $6 \%$ marginal $(n=18)$ and $85.7 \%(n=257)$ adequate health literacy level based on the S-TOFHLA reading comprehension passages. Regarding the numeracy items of the S-TOFHLA, $69.5 \%$ of participants answered correctly to item 1 (Correct
Table 1. Socio-demographic characteristics of the sample $(N=302)$

\begin{tabular}{|c|c|c|c|}
\hline $\begin{array}{l}\text { Socio- } \\
\text { demographic } \\
\text { characteristics }\end{array}$ & Variables & $\begin{array}{l}\text { Number of } \\
\text { participants }\end{array}$ & Percentage \\
\hline \multirow{2}{*}{ Gender } & Female & 160 & 53 \\
\hline & Male & 142 & 47 \\
\hline \multirow{5}{*}{ Marital status } & Married & 131 & 43 \\
\hline & Divorced & 42 & 14 \\
\hline & Widowed & 20 & 7 \\
\hline & Single & 57 & 19 \\
\hline & In a relationship & 52 & 17 \\
\hline \multirow{3}{*}{ Age groups } & $18-45$ & 145 & 48 \\
\hline & $46-65$ & 112 & 37 \\
\hline & $>65$ & 45 & 15 \\
\hline \multirow{3}{*}{ Education } & $\begin{array}{l}\text { Primary/vocational } \\
\text { school }\end{array}$ & 71 & 24 \\
\hline & $\begin{array}{l}\text { Secondary grammar } \\
\text { school/high school }\end{array}$ & 146 & 48 \\
\hline & College/university & 85 & 28 \\
\hline \multirow{3}{*}{$\begin{array}{l}\text { Income in } \\
\text { Hungarian Forint }\end{array}$} & 0-90,000 HUF & 111 & 37 \\
\hline & $91-200,000$ HUF & 119 & 39 \\
\hline & $>201,000$ HUF & 72 & 24 \\
\hline
\end{tabular}

medication taking), $85.1 \%$ for item 2 (Normal blood sugar level), 95\% for item 3 (Appointment card) and $83.8 \%$ for item 4 (Correct medication taking). The mean score was $30.63(\mathrm{SD}=7.67)$ of the reading comprehension passages and $3.34(\mathrm{SD}=0.86)$ of the numeracy items.

The Chew screening measure consists of three questions, responses were scored on a scale from 0 to 4 . The scores of item 1 were reversed. Then, the scores on the 3 items were added, therefore, the overall score ranged from 0 to 12 . The higher scores indicate a lower health literacy level. The mean score was 4.25 $(\mathrm{SD}=2.5)$.

\section{Association between Health Literacy Tests and Their Relationship with Socio-demographic Variables}

In order to examine the convergent validity, we tested the Spearman's rho correlations between the Hungarian-version of the S-TOFHLA, the Chew screening questions and the NVS. The S-TOFHLA reading comprehension part showed a strong positive association with the NVS, and a positive moderate relationship with the S-TOFHLA numeracy part and a weak negative correlation with the Chew questions. The NVS showed a moderate positive relationship with the numeracy part of the S-TOFHLA. There was a weak negative association between the Chew questions and the NVS (Table 2).

Another important approach to examine construct validity is to examine the association between health literacy and sociodemographic variables. We tested the associations in case of the S-TOFHLA, in which the scores ranged from 0 (lowest health literacy) to 36 (highest health literacy) and the Chew screening questions (ranging between 0-12). 
Table 2. Correlations between health literacy tests

\begin{tabular}{|l|c|c|c|}
\hline Measure & 1 & 2 & 3 \\
\hline S-TOFHLA reading comprehension & - & & \\
\hline S-TOFHLA numeracy & $0.442^{* *}$ & - & \\
\hline Chew questions & $-0.280^{* *}$ & $-0.114^{*}$ & - \\
\hline Newest Vital Sign & $0.605^{* *}$ & $0.443^{* *}$ & $-0.284^{* *}$ \\
\hline
\end{tabular}

${ }^{*} \mathrm{p}<0.05,{ }^{* *} \mathrm{p}<0.01$

In case of the S-TOFHLA and Newest Vital Sign higher scores correspond to a higher level of health literacy. The negative associations with the Chew questions are due to the inverted coding of this scale, the higher scores indicating lower health literacy.

First, three age groups were defined $(8,10)$, and the mean variances were tested using one-way ANOVA. The younger participants achieved significantly higher scores in the S-TOFHLA reading comprehension part, and the LSD posttest revealed a significant difference among all three groups. Younger respondents also showed a tendency to report fewer self-perceived health literacy problems in the Chew questions, but the difference reached significance only between the youngest and oldest age group $(p=0.02)$.

Second, based on the educational background, we divided our sample into three groups: participants with primary or vocational school degree, participants with secondary grammar school or high school education, and finally university degree. The ANOVA results confirmed that highly educated people possess higher levels of health literacy, both in case of the S-TOFHLA and the Chew questions. The LSD posttest showed significant differences between the three groups.

Third, participants with higher income also achieved higher health literacy scores in the S-TOFHLA, although there was a significant difference only between the participants with the highest income compared to the low $(p<0.01)$ and middle-income group $(p=0.01)$ based on the ANOVA LSD posttest. There was no difference in the Chew screening questions between the three income groups (Table 3).

Fourth, participants living with a chronic condition achieved significantly lower health literacy scores in the S-TOFHLA reading comprehension section compared to the healthy respondents based on the results of the independent samples t-test (28). Additionally, chronically ill participants also reported more selfperceived health literacy related problems in the Chew screening questions.

Finally, female respondents achieved significantly higher scores in the S-TOFHLA reading comprehension section than males compared with independent samples t-test. Similarly, there was a tendency that women report less self-perceived difficulties in the medical context in the Chew screening questions than men (Table 4).

In a linear regression model, age and education were significant predictors, explaining $47.1 \%$ of the health literacy level based in the S-TOFHLA reading comprehension section $(\mathrm{F}(2 ; 299)=$ 134.267, $\mathrm{p}<0.001)$.

\section{DISCUSSION}

In our sample, $14.3 \%$ of the respondents had either inadequate or marginal health literacy $(8.3 \%$ inadequate, $6 \%$ marginal health literacy scores). Our results are in accordance with the European outcomes of the S-TOFHLA, as in many countries the proportion of either marginal or inadequate health literacy was around 10 to $15 \%$. For instance, in a study measuring the prevalence of limited functional health literacy in the UK, this proportion was $11.4 \%$ (17). The health literacy level in Hungary is the most similar to the outcomes of the French-speaking Swiss population (8). Moreover, based on the HLS-EU survey around $12.4 \%$ of the respondents showed inadequate health literacy, and further $35 \%$ problematic health literacy on average in the participating countries (15). In Hungary, based on the HLS-EU survey, 20\% of the respondents possessed inadequate and $32 \%$ problematic health literacy $(20,21)$, which is much higher than the $8.3 \%$ inadequate and $6 \%$ marginal health literacy proportions found in our study. This difference might be explained by the difference in the operationalization of health literacy between the studies: while the S-TOFHLA objectively measures functional health literacy, the HLS-EU survey is based on self-reported data and focuses on the cultural aspects and critical skills of health literacy (28).

Table 3. Association between age, education, income groups and health literacy

\begin{tabular}{|c|c|c|c|c|c|c|c|c|}
\hline & \multicolumn{4}{|c|}{ S-TOFHLA reading comprehension } & \multicolumn{4}{|c|}{ Chew screening questions } \\
\hline & Mean & SD & $\mathbf{F}$ & $\mathrm{p}$-value & Mean & SD & $F$ & $\mathrm{p}$-value \\
\hline \multicolumn{9}{|l|}{ Age groups } \\
\hline $18-45$ & 34.32 & 3.07 & \multirow{3}{*}{85.18} & \multirow{3}{*}{$<0.001$} & 3.93 & 2.08 & \multirow{3}{*}{2.991} & \multirow{3}{*}{0.052} \\
\hline $46-65$ & 29.76 & 6.98 & & & 4.38 & 2.82 & & \\
\hline$>65$ & 20.68 & 10.18 & & & 4.93 & 2.76 & & \\
\hline \multicolumn{9}{|l|}{ Education } \\
\hline Primary/vocational school & 23.45 & 9.80 & \multirow{3}{*}{58.49} & \multirow{3}{*}{$<0.001$} & 5.87 & 2.46 & \multirow{3}{*}{29.66} & \multirow{3}{*}{$<0.001$} \\
\hline Secondary grammar school/high school & 32.21 & 5.09 & & & 4.15 & 2.27 & & \\
\hline College/university & 34.00 & 5.10 & & & 3.04 & 2.17 & & \\
\hline \multicolumn{9}{|l|}{ Income in Hungarian Forint } \\
\hline 0-90,000 HUF & 28.81 & 8.72 & \multirow{3}{*}{8.537} & \multirow{3}{*}{$<0.001$} & 4.52 & 2.50 & \multirow{3}{*}{1.737} & \multirow{3}{*}{0.178} \\
\hline $91-200,000$ HUF & 30.60 & 7.49 & & & 4.25 & 2.44 & & \\
\hline$>201,000$ HUF & 33.50 & 4.97 & & & 3.81 & 2.57 & & \\
\hline
\end{tabular}


Table 4. Association between gender, presence of a chronic condition and health literacy

\begin{tabular}{|c|c|c|c|c|c|c|c|c|c|c|}
\hline & \multicolumn{5}{|c|}{ S-TOFHLA reading comprehension } & \multicolumn{5}{|c|}{ Chew screening questions } \\
\hline & Mean & SD & $\mathrm{t}$ & df & $\mathrm{p}$-value & Mean & SD & $\mathrm{t}$ & df & $\mathrm{p}$-value \\
\hline \multicolumn{11}{|c|}{ Chronic condition } \\
\hline Yes & 26.52 & 9.64 & \multirow{2}{*}{7.105} & \multirow{2}{*}{$147.7^{\AA}$} & \multirow{2}{*}{$<0.001$} & 5.11 & 2.56 & \multirow{2}{*}{-4.98} & \multirow{2}{*}{300} & \multirow{2}{*}{$<0.001$} \\
\hline No & 33.26 & 4.43 & & & & 3.69 & 2.30 & & & \\
\hline \multicolumn{11}{|l|}{ Gender } \\
\hline Male & 28.99 & 8.85 & \multirow{2}{*}{-3.46} & \multirow{2}{*}{$243.23^{A}$} & \multirow{2}{*}{0.001} & 4.51 & 2.68 & \multirow{2}{*}{1.731} & \multirow{2}{*}{$279.83^{A}$} & \multirow{2}{*}{0.085} \\
\hline Female & 32.08 & 6.15 & & & & 4.01 & 2.30 & & & \\
\hline
\end{tabular}
therefore we used those versions which assume unequal variances.

Regarding the reliability of the instruments, the internal consistency of the Hungarian version of the S-TOFHLA reading comprehension section is excellent, while the numeracy part had a low internal consistency. The Chew screening questions had adequate reliability scores.

The strong link between the S-TOFHLA and the NVS provides evidence of the construct validity. The Chew screening questions showed only a weak association with the S-TOFHLA and the NVS, similarly as in the Turkish study (12). The two tests might measure different segments of health literacy (28), or the Chew screening questions might have social desirability bias (23). Yet, the Chew screening measure remains a useful tool primarily in the clinical context, as it provides a quick proxy for the patient's self-perceived difficulties concerning their functional health literacy skills. However, the outcomes of the Chew screening measure have to be interpreted with caution, as people tend to be overconfident in rating their own knowledge and skills (29).

Regarding the socio-demographic predictors, our results show that older participants possess a lower level of health literacy, which is in accordance with previous findings $(8,10,11,15)$. Similarly, respondents with lower educational attainment are more probable to achieve lower scores, which correspond with the findings of other validation studies as well as the outcomes of the HLS-EU survey $(8,10,11,15)$. Income was one of the socio-demographic determinants of health literacy $(10,11)$. However, it was a less stable predictor, as only participants with the highest income achieved significantly higher S-TOFHLA reading comprehension scores than respondents with income in the middle or low range. There was no difference based on the income in the Chew screening measure. Women possessed a higher level of health literacy $(16,17)$. Finally, chronically ill respondents had significantly lower health literacy scores (28). An association between health literacy and chronic condition has been also suggested in the HLS-EU survey as well as previous research in Hungary $(15,20,21)$. As chronically ill needs to use and understand health-related information on a regular basis, it is crucial to increase the health literacy level in this population.

\section{CONCLUSIONS}

The Hungarian version of the S-TOFHLA has been shown to be a valid and reliable measure of functional health literacy. However, we would advise to use only the reading comprehen- sion part of the S-TOFHLA for the time being. As the numerical part showed a low internal consistency, further research is needed on its psychometric properties before its practical application. Moreover, the Hungarian version of the Chew screening questions provides a valid self-reported assessment, which we recommend to use for the detection of patients with inadequate health literacy rapidly in the clinical setting. The translated text of the tools and the descriptive statistics are available in Hungarian language (28). The availability of these measures not only promotes further health literacy research in Hungary and developing health promotion policies, but also offers a useful tool to identify people with limited health literacy in the clinical context. Besides, minority groups (e.g. Roma people) report poor health status, unhealthy diet and smoking in Hungary (30). We propose that further research is needed to measure health literacy in this population, which can help us develop health education and campaigns reducing potential health disparities.

\section{Acknowledgements}

First, we thank to the participants of our study and the students who helped us in the data collection. Special thanks go to the leaders of the institutes helping with the data collection: Bíró Orsolya, Dr. Bálint András and Dr. Varga János.

\section{Conflict of Interests}

None declared

\section{Adherence to Ethical Standards}

Ethical approval was obtained from the Psychology Ethical Committee of the Universities in Hungary (2015/14) and the ethical committee of the hospital (1650_1/2015).

\section{REFERENCES}

1. Ratzan SC, Parker RM. Introduction. In: Selden CR, Zorn M, Ratzan SC, Parker RM, editors. Health literacy. Current bibliographies in medicine, no. 2000-1. Bethesda: National Library of Medicine; 2000. p. V-VII.

2. Berkman ND, Dewalt DA, Pignone MP, Sheridan SL, Lohr KN, Lux L, et al. Literacy and health outcomes. Evid Rep Technol Assess (Summ). 2004 Jan;(87):1-8.

3. Zhang NJ, Terry A, McHorney CA. Impact of health literacy on medication adherence: a systematic review and meta-analysis. Ann Pharmacother. 2014 Jun 1;48(6):741-51.

4. Williams MV, Baker DW, Honig EG, Lee TM, Nowlan A. Inadequate literacy is a barrier to asthma knowledge and self-care. Chest. 1998 Oct;114(4):1008-15. 
5. Davis TC, Long SW, Jackson RH, Mayeaux EJ, George RB, Murphy PW, et al. Rapid estimate of adult literacy in medicine: a shortened screening instrument. Fam Med. 1993 Jun;25(6):391-5.

6. Parker RM, Baker DW, Williams MV, Nurss JR. The test of functional health literacy in adults: a new instrument for measuring patients' literacy skills. J Gen Intern Med. 1995 Oct;10(10):537-41

7. Baker DW, Williams MV, Parker RM, Gazmararian JA, Nurss J. Development of a brief test to measure functional health literacy. Patient Educ Couns. 1999 Sep;38(1):33-42.

8. Connor M, Mantwill S, Schulz PJ. Functional health literacy in Switzerland - validation of a German, Italian, and French health literacy test. Patient Educ Couns. 2013 Jan;90(1):12-7.

9. Carthery-Goulart MT, Anghinah R, Areza-Fegyveres R, Bahia VS, Brucki SM, Damin A, et al. Performance of a Brazilian population on the test of functional health literacy in adults. Rev Saude Publica. 2009 Aug;43(4):631-8

10. Baron-Epel O, Balin L, Daniely Z, Eidelman S. Validation of a Hebrew health literacy test. Patient Educ Couns. 2007 Jul;67(1-2):235-9.

11. Jovic-Vranes A, Bjegovic-Mikanovic V, Marinkovic J. Functional health literacy among primary health-care patients: data from the Belgrade pilot study. J Public Health (Oxf). 2009 Dec;31(4):490-5.

12. Eyüboğlu E, Schulz PJ. Validation of Turkish health literacy measures. Health Promot Int. 2016 Jun;31(2):355-62

13. Tsai TI, Lee SY, Tsai YW, Kuo KN. Methodology and validation of health literacy scale development in Taiwan. J Health Commun. 2011 Jan;16(1):50-61.

14. Chew LD, Griffin JM, Partin MR, Noorbaloochi S, Grill JP, Snyder A, et al. Validation of screening questions for limited health literacy in a large VA outpatient population. J Gen Intern Med. 2008 May;23(5):561-6.

15. Sørensen K, Pelikan JM, Röthlin F, Ganahl K, Slonska Z, Doyle G, et al. Health literacy in Europe: comparative results of the European health literacy survey (HLS-EU). Eur J Public Health. 2015 Dec;25(6):1053-8

16. Parikh NS, Parker RM, Nurss JR, Baker DW, Williams MV. Shame and health literacy: the unspoken connection. Patient Educ Couns. 1996 Jan;27(1):33-9.

17. Von Wagner C, Knight K, Steptoe A, Wardle J. Functional health literacy and health-promoting behaviour in a national sample of British adults. J Epidemiol Community Health. 2007 Dec;61(12):1086-90.

18. Kondilis BK, Kiriaze IJ, Athanasoulia AP, Falagas ME. Mapping health literacy research in the European Union: a bibliometric analysis. PLoS One. 2008 Jun 25;3(6):e2519. doi: 10.1371/journal.pone.0002519.
19. Sørensen K, Van den Broucke S, Fullam J, Doyle G, Pelikan J, Slonska Z, et al; (HLS-EU) Consortium Health Literacy Project European. Health literacy and public health: a systematic review and integration of definitions and models. BMC Public Health. 2012 Jan 25;12:80. doi: 10.1186/1471-2458-12-80

20. Kun E. Measuring the prevalence of health literacy in European countries achievements and challenges - Hungary. In: 3rd European Health Literacy Conference; 2015 Nov 17-19; Brussels, Belgium.

21. Kun E. Health literacy: What does the HLS-EU measure? Reflecting on the Hungarian results considering the international outcomes. In: Conference on measuring and improving health literacy; 2016; Szeged, Hungary. (In Hungarian.)

22. Weiss BD, Mays MZ, Martz W, Castro KM, DeWalt DA, Pignone MP, et al. Quick assessmnet of literacy in primary care: the newest vital sign. Ann Fam Med. 2005 Nov-Dec;3(6):514-22.

23. Crowne DP, Marlowe D. A new scale of social desirability independent of psychopathology. J Consult Psychol. 1960 Aug;24:349-54.

24. Beaton DE Bombardier C, Guillemin F, Ferraz MB. Guidelines for the process of cross-cultural adaptation of self-report measures. Spine (Phila Pa 1976). 2000 Dec 15;25(24):3186-91.

25. Scholz U, Gutiérrez Doña B, Sud S, Schwarzer R. Is general self-efficacy a universal construct? Eur J Psychol Assess. 2002;18(3):242-51.

26. Spreitzer GM. Psychological empowerment in the workplace: dimensions, measurement, and validation. Acad Manage J. 1995 Oct;38(5):1442-65.

27. Mayer K, Lukács A, Pauler G. Hungarian adaptation of the 8-item Sensasition Seeking Scale. Mentálhigiéné és Pszichoszomatika. 2012 Sep;13(3):297-312. (In Hungarian.)

28. Papp-Zipernovszky O, Náfrádi L, Schulz PJ, Csabai M. "So each patient comprehends": measuring health literacy in Hungary. Orv Hetil. 2016 Jun;157(23):905-15. (In Hungarian.)

29. Alpert M, Raiffa H. A progress report on the training of probability assessors. In: Kahneman D, Slavic P, Tversky A, editors. Judgment under uncertainty: heuristics and biases. Cambridge: Cambridge University Press; 1982. p. 294-305.

30. Kósa Z, Széles G, Kardos L, Kósa, K, Németh R, Országh S, et al. A comparative health survey of the inhabitants of Roma settlements in Hungary. Am J Public Health. 2007 May; 97(5):853-9.

Received July 11, 2016

Accepted in revised form September 16, 2019 